\title{
Feasibility of portal or superior mesenteric vein resection and reconstruction by allogeneic vein for pancreatic head cancer-a case-control study
}

Xing-mao Zhang ${ }^{1+}$, Jie Zhang ${ }^{2+}$, Hua Fan ${ }^{1}$, Qiang He ${ }^{1}$ and Ren Lang ${ }^{1 *}$

\begin{abstract}
Background: There are few reports about resection of portal vein (PV)/superior mesenteric vein (SMV) and reconstruction by using allogeneic vein. This case-control study was designed to explore the feasibility and safety of this operation type in patients with T3 stage pancreatic head cancer.

Methods: A total of 42 patients (Group A) underwent PV/SMV resection and reconstruction by using allogeneic vein were 1:1 matched to 42 controls (Group B) with other types of resection and reconstruction. The two groups were well matched.

Results: There was no significantly prolonged total operation time (Group A vs. Group B [490.0 min vs. $470 \mathrm{~min}]$,

$P=0.067$ ) and increased intraoperative blood loss (Group A vs. Group B [650.0 min vs. $450 \mathrm{~min}], P=0.108$ ) was found between the two groups. R1 rate of PV/SMV was slightly reduced in group A compared to group B $(4.8 \%$ vs. $14.3 \%$,

$P=0.137)$, although no significant difference was found. The incidences of main postoperative complications between the two groups were similar. A slightly increased 1-year and 2-year overall survival rate (OS) (Group A vs. Group B [1-year OS: $62.9 \%$ vs. 57.0\%; 2-year OS: $31.5 \%$ vs. 25.6\%], $P=0.501$ ) and disease-free survival rate (DFS) (Group A vs. Group B [1-year DFS: 43.9\% vs. 36.6\%; 2-year DFS: 10.5\% vs. 7.4\%], $P=0.502$ ) could be found in group A compared to group $B$, although the differences were not significant.
\end{abstract}

Conclusions: The operation types of PV/SMV resection and reconstruction by using allogeneic vein is safety and feasible, it might have a potential benefit for patients.

Keywords: Pancreatic cancer, Vascular resection, Reconstruction, Outcome

\section{Background}

With increasing incidence and mortality, cancer is the one of leading causes of death worldwide and it has become a major public problem. Among the malignant solid tumors, pancreatic cancer is characterized by low curative rate and high mortality [1-5]. Cancer Statistics in China which is completed by National Cancer Center

\footnotetext{
*Correspondence: langren_chaoyang@163.com

${ }^{\dagger}$ Equal contributors

${ }^{1}$ Department of hepatobiliary surgery, Beijing Chaoyang Hospital, Capital Medical University, 8 Gongti South Street, Chaoyang District, Beijing 100021 China

Full list of author information is available at the end of the article
}

of China shows that the estimated new cancer cases and deaths of pancreas are 90.1 and 79.4 thousand in 2015 [6]. Despite decades of effort, pancreatic cancer remains one of the most aggressive and lethal malignancies, and the five-year survival rate remains at only 5\% [7]. Due to biological characteristics of pancreatic cancer, also due to anatomical location of pancreas and lack of early detection tests, most of patients with localized disease have no recognizable symptoms or signs. As a result, only 15 to $25 \%$ of patients with pancreatic cancer is eligible for curative resection. Of the unresectable patients, approximately two thirds present with distant metastases

(c) The Author(s). 2018 Open Access This article is distributed under the terms of the Creative Commons Attribution 4.0 International License (http://creativecommons.org/licenses/by/4.0/), which permits unrestricted use, distribution, and reproduction in any medium, provided you give appropriate credit to the original author(s) and the source, provide a link to the Creative Commons license, and indicate if changes were made. The Creative Commons Public Domain Dedication waiver (http://creativecommons.org/publicdomain/zero/1.0/) applies to the data made available in this article, unless otherwise stated. 
and the rest presents with locally advanced disease which has surrounding vascular invasion. According to the TNM staging system of pancreatic cancer, tumor extending to portal vein (PV) or superior mesenteric vein (SMV) without involvement of celiac axis or superior mesenteric artery is defined as T3 stage. Based on performance status and preference of patients, and decision of Multi-Disciplinary Team, different therapeutic modalities including upfront surgery, neoadjuvant chemotherapy followed by surgery, chemotherapy alone, chemoradiotherapy, etc., can be selected for T3 stage pancreatic cancer which is clinically diagnosed. Of these modalities, upfront surgery is most frequently used.

Radical pancreatic surgery accompanied with PV/SMV resection, has commonly been undertaken for pancreatic cancer with infiltration of PV/SMV since the first report of pancreatoduodenectomy (PD) with resection and reanastomosis of vein in $1951[8,9]$. Till now, there has been no consensus yet on the survival advantage of PV/ SMV resection and reconstruction for pancreatic head cancer, although similar reported short-term outcomes when compared with standard PD [10-13]. In the classification of International Study Group of Pancreatic Surgery (ISGPS), a total of four types of venous reconstruction are included [14]. With the view to improving the R0 resection rate, to reaching tension-free anastomosis, to avoiding the diameter mismatch between the PV and SMV, and also to avoiding vessel stenosis, segmental resection and reconstruction by using allogeneic vein has been preferred for selected patients since 2013 in our center.

In this study, we conducted a case-control study to explore the feasibility and safety of PV/SMV resection and reconstruction by using allogeneic vein in patients with pancreatic head adenocarcinoma.

\section{Methods \\ Patients}

From January 2012 to November 2016, patients with pancreatic head cancer who underwent surgical treatment at our institution were reviewed from our prospectively collected clinical database. Clinicopathological data of patients with duct adenocarcinoma of pancreatic head with PV/SMV infiltration by postoperative pathology were used for analysis. Patients with advanced pancreatic cancer, or with $\mathrm{T} 4$ stage pancreatic cancer, or without PV/SMV infiltration were excluded from this study, neither patients with cancer history nor patients with neoadjuvant therapy were enrolled in this study.

Patients underwent segmental PV/SMV resection with reconstruction using allogeneic vein were 1:1 matched with patients underwent PV/SMV resection with reconstruction by other methods (direct end-to-end anastomosis, partial venous excision with direct closure, partial venous excision using a patch or artificial vascular graft).
Matching criteria included age, gender, body mass index (BMI), American Society of Anesthesiology (ASA) score, concomitant disease, blood test, surgical procedures (extended PD with extended Lymphadenectomy), postoperative pathology, and adjuvant therapy (Table 1). When investigators did the manual match, they were blinded to the surgical outcomes to reduce potential selection bias.

\section{Surgical modality}

In our center, four types of venous invasion were concluded. Type one, solid tumor contact with the PV/SMV of $\leq 90^{\circ}$; type two, solid tumor contact with the PV/SMV of $>90^{\circ}$, or obvious stenosis or occlusion of $\mathrm{PV} / \mathrm{SMV}$, without extension to confluence of splenic vein; type three, involvement of superior mesenteric-portal-splenic vein confluence; type four, involvement of both of superior mesenteric-portal-splenic vein confluence and branches of superior mesenteric vein. Patients with type one of PV/SMV invasion received partial venous excision with direct closure or partial venous excision using a patch, and patients with type two can received segmental PV/SMV resection with direct end-to-end anastomosis (length of invasion $\leq 2 \mathrm{~cm}$ ) or reconstruction using allogeneic vein (length of invasion $>2 \mathrm{~cm}$ ), whereas for patients with type three or type four, only segmental PV/SMV resection with reconstruction using allogeneic vein can be performed (Vascular reconstruction was completed for pancreatic head cancer with different type of venous invasion, as shown in Fig. 1).

According to the guidelines of ISGPS, standard PD combined with excision of PV/SMV could be defined as extended PD, and all patients underwent extended PD in this study; extended lymphadenectomy including the removal of lymph nodes of stations $5,6,8 \mathrm{a}, 8 \mathrm{p}, 9,12 \mathrm{a}$, 12p, 12b1, 12b2, 12c, 13a-b, 14a-d, 16a2, 16b1, and $17 \mathrm{a}-\mathrm{b}$ was applied for all patients.

\section{Postoperative management}

Subcutaneous injection of nodroparin calcium $0.4 \mathrm{~mL}$ per day was started on the second day after operation and ended on the seventh days, then 1 month of oral aspirin was recommended. Doppler B-ultrasound was used for observing the venous blood flow on the third and seventh day after operation. Computed tomographic angiography (CTA) was used for justifying the condition of PV/SMV 1 month after operation, and then 6 months postoperatively; meanwhile Doppler was also used for evaluation of PV/SMV blood flow.

\section{Parameters for analysis}

Demographic and clinicopathologic parameters, operative details, perioperative complications and prognosis recorded to our database were used for analyzed. Drain amylase of $>3$ times serum amylase after the third 
Table 1 General parameters of the two groups

\begin{tabular}{|c|c|c|c|}
\hline Parameters & Group A $(n=42)$ & Group B $(n=42)$ & $P$-value \\
\hline Gender, case (\%) & & & 0.826 \\
\hline Male & $18(42.9)$ & $19(45.2)$ & \\
\hline Female & $24(57.1)$ & $23(54.8)$ & \\
\hline Age, year, median (range) & $68(43-80)$ & $67(45-79)$ & 0.952 \\
\hline BMI, kg/m², median, (range) & $21.5(17.6-28.6)$ & $23.1(17.3-29.7)$ & 0.238 \\
\hline ASA Score (\%) & & & 0.608 \\
\hline 2 & $11(26.2)$ & $9(21.4)$ & \\
\hline 3 & $31(73.8)$ & $33(78.6)$ & \\
\hline Concomitant disease, case (\%) & $32(76.2)$ & $33(78.6)$ & 0.794 \\
\hline Abdominal operation history, case (\%) & $4(9.5)$ & $3(7.1)$ & 0.693 \\
\hline Preoperative biliary drainage, case (\%) & $8(19.0)$ & $7(16.7)$ & 0.776 \\
\hline Hemoglobin, g/L, median (range) & $112.5(86-153)$ & $112.0(87-151)$ & 0.673 \\
\hline Total bilirubin, $\mu$ mol//, median (range) & $84.9(10.1-440)$ & $79.4(19.9-423)$ & 0.989 \\
\hline Direct bilirubin, $\mu \mathrm{mol} / \mathrm{I}$, median (range) & $64.1(6.3-347)$ & $69.8(12.8-367)$ & 0.733 \\
\hline Carbohydrate antigen 19-9, U/ml, median (range) & $511.5(2.0-6021)$ & $493.4(2.1-5966)$ & 0.834 \\
\hline Tumor size, cm, median (range) & $3.5(2.0-5.0)$ & $3.3(2.0-5.0)$ & 0.557 \\
\hline Tumor differentiation, case (\%) & & & 0.903 \\
\hline Well & $2(4.8)$ & $2(4.8)$ & \\
\hline Moderate & $24(57.1)$ & $22(52.4)$ & \\
\hline Poor & $16(38.1)$ & $18(42.9)$ & \\
\hline N stage, case (\%) & & & 0.728 \\
\hline NO & $4(9.5)$ & $5(11.9)$ & \\
\hline N1 & $38(90.5)$ & $37(88.1)$ & \\
\hline Adjuvant therapy & & & 0.977 \\
\hline None & $4(9.5)$ & $5(11.9)$ & \\
\hline Gemcitabine & $14(33.3)$ & $12(28.6)$ & \\
\hline Gemcitabine+ Capecitabine & $5(11.9)$ & $6(14.3)$ & \\
\hline S1 & $12(28.6)$ & $11(26.2)$ & \\
\hline FOLFIRINOX & $7(16.7)$ & $8(19.0)$ & \\
\hline
\end{tabular}

ASA, American Society of Anesthesiology; BMI, body mass index; FOLFIRINOX, leucovorin and fluorouracil plus irinotecan and oxaliplatin

postoperative day, as defined by ISGPS [15], was defined as pancreatic fistula. The status of resection margins was classified based on the International Union Against Cancer R classification, as microscopic radical resection (R0), microscopic positive (R1), or gross positive (R2). Margin $<1 \mathrm{~mm}$ was defined as positive margin. Postoperative complications were monitored for 30 days after surgery, and perioperative mortality was defined as death within 30 days after surgery or during hospital stay.

\section{Follow-up and statistical analysis}

Physical examination, laboratory tests, and image examinations were used for follow-up, the follow-up interval was 2 months. SPSS 16.0 (IBM, Chicago, Illinois, USA) was used for data analysis. Quantitative variables were expressed as median and range, and comparisons were analyzed with the Student t-test according to data distribution. Categorical variables were presented as number and percentage and were analyzed by chi-square test or Fisher exact test, as appropriate. Kaplan-Meier method was used to analyze the survival of patients, and the curve of survival between groups was analyzed by logrank test.

\section{Results}

As shown in Fig. 2, from January 2012 to November 2016, a total of 348 patients underwent extended PD in our hospital. Of these patients, 269 cases were confirmed as duct adenocarcinoma of pancreatic head by postoperative pathology. According to the TNM staging of pancreatic cancer of American Joint Committee on Cancer (AJCC), 184 cases had the T3 stage duct 

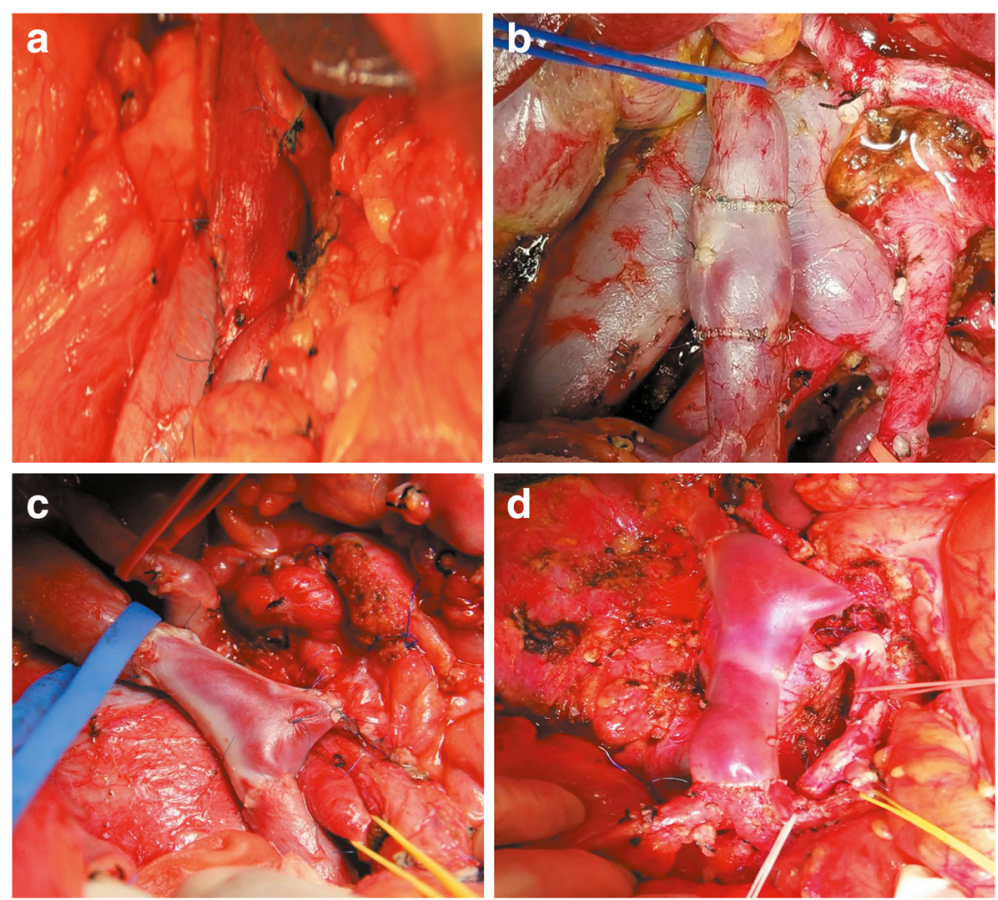

Fig. 1 Four types of PV/SMV invasion for pancreatic head cancer. Different reconstruction modality for different type of vascular invasion was presented. a Tumor contacted with the PV/SMV of $\leq 90^{\circ}$, and partial venous excision with direct closure was performed; b-d Segmental PV/SMV (including superior mesenteric-portal-splenic vein confluence in figure c, and both superior mesenteric-portal-splenic vein confluence and partial first jejunal branch in figure d) resection with reconstruction using allogeneic vein was performed: $\mathbf{b}$ Tumor contacted with the PV/SMV of $>90^{\circ}$ and the length of invasion was $>2 \mathrm{~cm}$. c Superior mesenteric-portal-splenic vein confluence was involved; d Both of superior mesenteric-portalsplenic vein confluence and branches of superior mesenteric vein (first jejunal branch) were involved

adenocarcinoma. 42 received segmental PV/SMV resection with reconstruction using allogeneic vein in all of the 184 patients. Of the 42 patients, type $2 \mathrm{PV} / \mathrm{SMV}$ invasion was confirmed in 32 cases, type three in 8 cases, and type four in 2 cases according to our classification of venous invasion (group A).

Patients with reconstruction using allogeneic vein (group A) were 1:1 matched to patients with reconstruction by other methods including segmental excision with direct end-to-end anastomosis, partial venous excision with direct closure, partial venous excision using a patch and segmental excision with artificial vascular graft (group B). In group B, type 1 $\mathrm{PV} / \mathrm{SMV}$ invasion was confirmed in 17 patients and type two in 25 patients according to our classification of venous invasion. Of the 42 patients, 13 cases received partial venous excision with direct closure, four cases received partial venous excision using a patch, 22 cases received segmental excision with direct end-to-end anastomosis, and three cases received segmental excision with artificial vascular graft. Demographics and clinical characteristics are shown in Table 2. The two groups were well balanced in terms of age, gender, BMI, ASA score, concomitant disease, blood test, postoperative pathology, and adjuvant therapy (Table 1).

As shown in Table 2, there was no statistically significant difference was found for operation time between the two groups (490 $\mathrm{min}$ vs. $470 \mathrm{~min}, P=0.067$ ), although the time to vein reconstruction in group A was longer compared with that in group B (52.5 min vs. $45 \mathrm{~min}, P<0.001)$. The volume of intraoperative blood loss was $650 \mathrm{~mL}$ and $450 \mathrm{~mL}$ in group $\mathrm{A}$ and group $\mathrm{B}$, respectively $(P=0.108)$. The length of PV/SMV resected was measured in this study, and the median length was $3.0 \mathrm{~cm}$ in group A which was obviously longer than that in group B. Although no significant difference for the R1 rate of $\mathrm{PV} / \mathrm{SMV}$, the $\mathrm{R} 1$ rate decreased almost $10 \%$ in group A compared with group B (4.8\% vs. $14.3 \%)$. The incidences of pancreatic fistula were 14.3 and $19.0 \%$, no significant difference was found $(P=0.558)$, the same result could be found for incidence of bleed (4.8\% vs. $4.8 \%$ ). The stump bleeding of gastroduodenal artery was confirmed for all four patients. Emergency laparotomy was carried out for two patients, interventional embolization was used for another two patients, and none died from bleed. No portal vein thrombosis was found by doppler on the third and seventh day after operation 


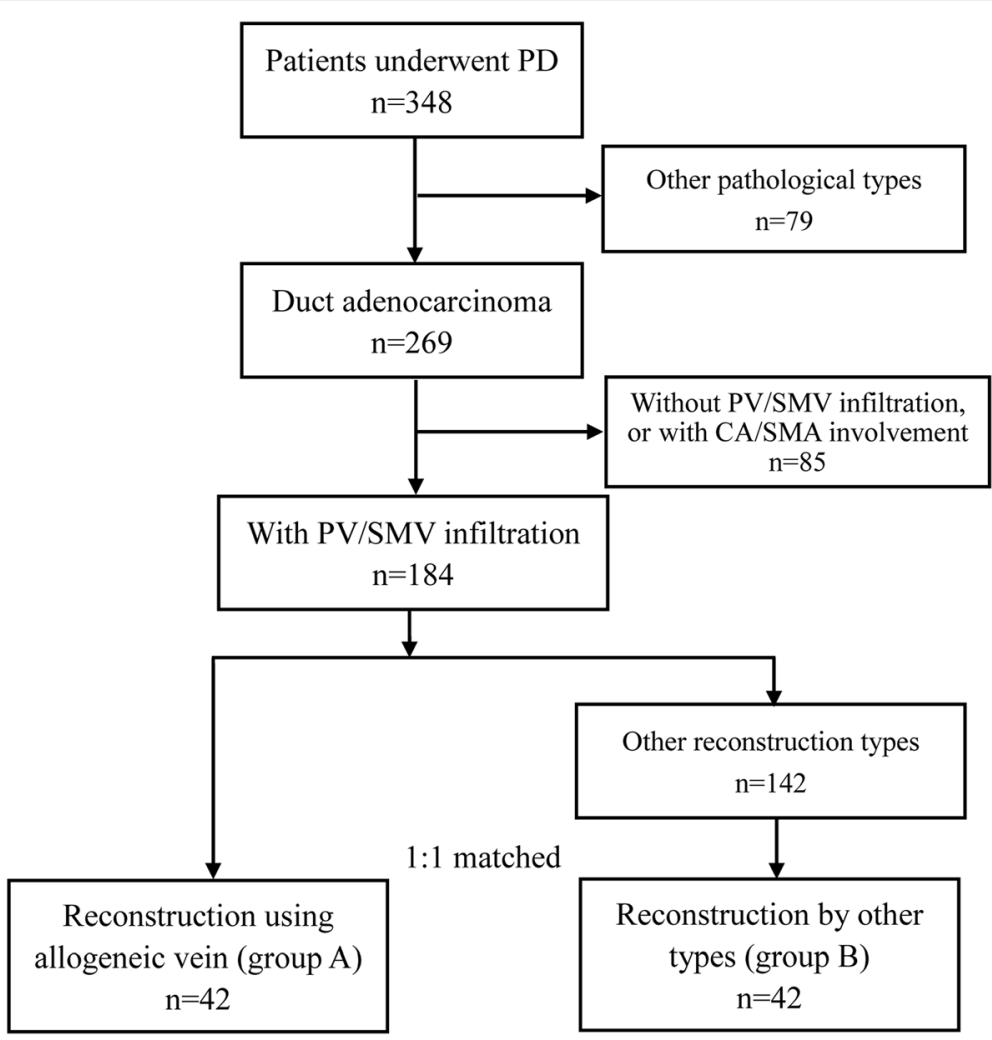

Fig. 2 The patients flow in this study

Table 2 Operation outcomes and long-term outcomes of the two groups

\begin{tabular}{|c|c|c|c|}
\hline Parameters & Group A $(n=42)$ & Group B $(n=42)$ & $P$-value \\
\hline Operation time, min, median (range) & $490.0(360.0-920.0)$ & $470(280.0-650.0)$ & 0.067 \\
\hline Time to vein reconstruction, min, median (range) & $52.5(40.0-80.0)$ & $45.0(30.0-70.0)$ & $<0.001$ \\
\hline Intraoperative blood loss, ml, median (range) & $650.0(350.0-1650.0)$ & $450.0(250.0-1350.0)$ & 0.108 \\
\hline Lymph node retrieved & $18.5(7.0-50.0)$ & $18.0(6.0-46.0)$ & 0.278 \\
\hline Length of vein resected, cm, median (range) & $3.0(2.0-6.0)$ & $2.0(1.0-4.0)$ & $<0.001$ \\
\hline R1 rate of $\mathrm{PV} / \mathrm{SMV}$ & $2(4.8)$ & $6(14.3)$ & 0.137 \\
\hline \multicolumn{4}{|l|}{ Postoperative complications, case (\%) } \\
\hline Pancreatic fistula & $6(14.3)$ & $8(19.0)$ & 0.558 \\
\hline Bleed & $2(4.8)$ & $2(4.8)$ & 1.000 \\
\hline Delayed gastric emptying & $5(11.9)$ & $4(9.5)$ & 0.724 \\
\hline Portal vein thrombosis & 0 & $1(2.4)$ & 0.314 \\
\hline OS, \% & & & 0.501 \\
\hline 1-year OS & 62.9 & 57.0 & \\
\hline 2-year OS & 31.5 & 25.6 & \\
\hline 3-year OS & 0 & 0 & \\
\hline DFS, \% & & & 0.502 \\
\hline 1-year DFS & 43.9 & 36.6 & \\
\hline 2-year DFS & 10.5 & 7.4 & \\
\hline
\end{tabular}


in group A, but one patient had portal vein thrombosis conformed by doppler B-ultrasound. There was no patient with infectious complication related to allogeneic vein.

As shown in Fig. 3, the 1- and 2-year OS were 62.9 and $31.5 \%$ in group $\mathrm{A}$, and 57.0 and $25.6 \%$ in group B $(P=0.501)$. The 1 - and 2-year DFS were 43.9 and $10.5 \%$ in group A, 36.6 and $7.4 \%$ in group $B$, respectively $(P=0.502)$, which were shown in Fig. 4 . Neither group A nor group B had 3-year OS.

\section{Discussion}

Radical resection is still the only potential method for the cure of pancreatic cancer although the development of non-surgical fields [16-18]. There is no unanimous consensus yet on the efficacy of vascular resection for pancreatic cancer with infiltration of PV/SMV [19-21]. Recently, the argument about the value of PV/SMV resection has become more and more prevalent [22]. As an example, a study designed by Murakami $\mathrm{Y}$ et al. [12]. Showed that PD with PV/SMV resection and adjuvant chemotherapy in patients with pancreatic head carcinoma might provide good survival without increased mortality and morbidity; whereas a meta-analysis designed by F. Giovinazzo et al. [10]. Revealed that inferior results including increased postoperative mortality, higher rates of non-radical surgery and worse survival were related to PV/SMV resection. Especially to deserve to be mentioned, these two articles were published in the same journal during a short interval. Although the widespread growth of controversy, PV/SMV resection and reconstruction has been still recommended by some centers $[9,22-25]$.

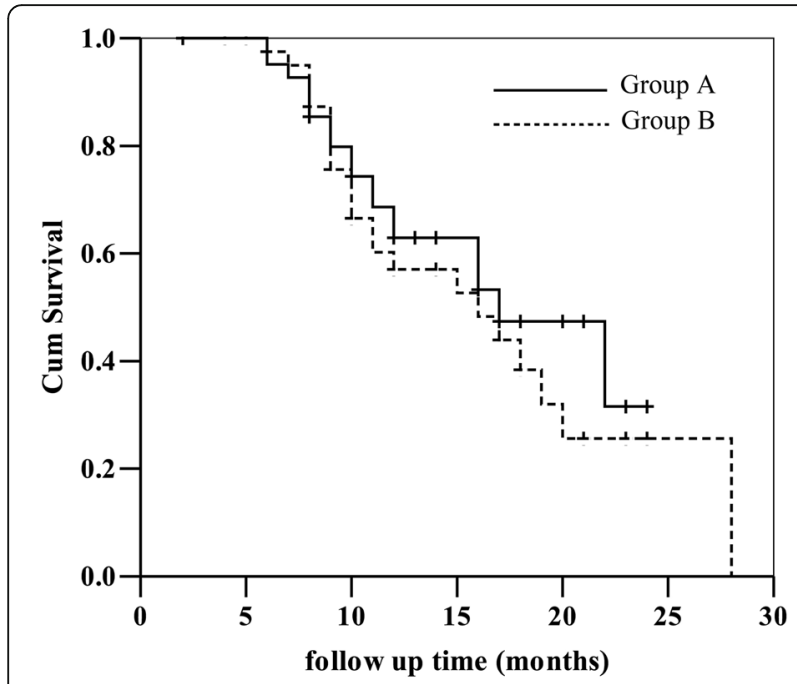

Fig. 3 The overall survival rate of the two groups

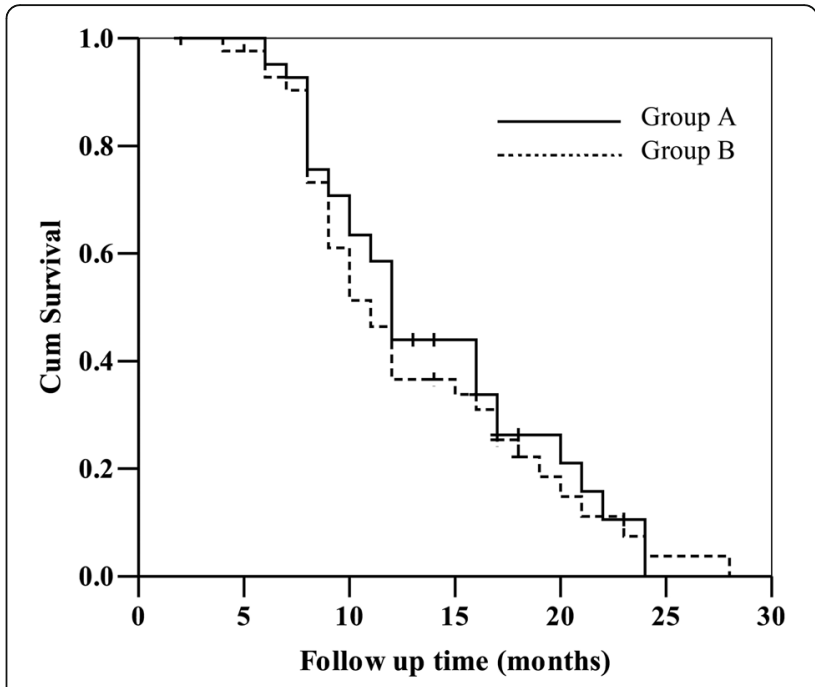

Fig. 4 the disease-free survival rate of the two groups

Four types of venous resection are recommended by ISGPS [14]: partial venous excision with direct closure (venorraphy) by suture closure; partial venous excision using a patch; segmental resection with primary venovenous anastomosis; segmental resection with interposed venous conduit and at least two anastomoses. We think that partial venous excision with direct closure or using a patch may be feasible when venous infiltration is less than $90^{\circ}$, whereas it is not suitable when involvement of $\mathrm{PV} / \mathrm{SMV}$ is more than $90^{\circ}$. Two main related drawbacks are vessel stenosis and increased R0 rate. We also suggest that segmental resection with direct venovenous anastomosis is preferred when length of invasion $\leq 2 \mathrm{~cm}$ with venous involvement $>90^{\circ}$, direct end-to-end anastomosis may not be the best choice if the length of venous invasion $>2 \mathrm{~cm}$. The disadvantages of end-toend anastomosis mainly includes increased tension of blood vessel and diameter mismatch. For some infiltration of special location such as superior mesentericportal-splenic vein confluence, neither direct end-to-end anastomosis nor artificial blood vessel is suitable. Venous resection and reconstruction by using allogeneic vein may provide an alternative way.

Compared with routine venous resection and reconstruction, the coexistence of advantages and disadvantages can be observed for allogeneic vein reconstruction. We conclude the main advantages of this technique: Firstly, extending surgical indications of pancreatic cancer. Without excessive limitation by infiltration scope of $\mathrm{PV} / \mathrm{SMV}$, patients have more chance to receive surgical resection. In our study, we could see that the length of $\mathrm{PV} / \mathrm{SMV}$ resected in group A was obviously longer than group B. Secondly, improving en-bloc resection rate and $\mathrm{R} 0$ rate, more patients can benefit from radical resection. 
In this study, R1 rate of PV/SMV in group A decreased by nearly $10 \%$ compared with that in group B although no significant difference. Thirdly, avoiding incidences of vascular tension after anastomosis, vascular stenosis, anastomotic stricture and diameter mismatch. Recovering normal anatomic structure of vascular for special location such as superior mesenteric-portal-splenic vein confluence to the most extent. Fourthly, avoiding rejection induced by artificial vascular and long-time anticoagulation treatment.

The main disadvantages of this technique are easily observed. Obviously, the procedure of allogeneic vein reconstruction is more complex compared with routine types, thus more operation time is needed. In this study, we could see that the time to vein reconstruction was significantly longer in group A than that in group B, whereas the total operation time and intraoperative blood loss between the two groups were comparable. Preservation of blood vessel is another issue of concern, there has been no unified standard till now.

The complications did not increase in patients underwent allogeneic vein reconstruction, and no increased vein-related complications were found; meanwhile, the prognosis in these patients had slight improvement although no significant difference.

\section{Conclusions}

PV/SMV resection and reconstruction by using allogeneic vein is safety and feasible, it may extend surgical indications and increase $\mathrm{R} 0$ resection rate compared with routine types.

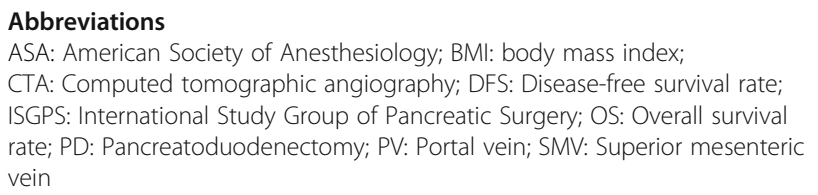

\section{Acknowledgements}

All authors thank Dai Yang and Zhang Xin-xue for supporting data, their supports are the key factor for completion of this manuscript.

\section{Availability of data and materials}

The datasets used and/or analysed during the current study available from the corresponding author on reasonable request.

\section{Authors' contributions}

LR and ZXM provided the concept, ZXM and ZJ were the major contributor in writing the manuscript, $\mathrm{HQ}$ and $\mathrm{FH}$ analyzed and interpreted the patient data, all authors read and approved the final manuscript.

\section{Ethics approval and consent to participate}

This study was reviewed and approved by the Ethics Committee of Beijing Chaoyang Hospital. Patients were not required to give informed consent to the study because the analysis used anonymous clinical data that were obtained after each patient agreed to treatment by written consent.

\section{Competing interests}

The authors declare that they have no competing interests.

\section{Publisher's Note}

Springer Nature remains neutral with regard to jurisdictional claims in published maps and institutional affiliations.

\section{Author details}

'Department of hepatobiliary surgery, Beijing Chaoyang Hospital, Capital Medical University, 8 Gongti South Street, Chaoyang District, Beijing 100021, China. ${ }^{2}$ The First Hospital of Combination of the Western Medicine and Traditional Chinese Medicine, Xiaozhuang Hospital, Capital Medical University, 13 Jintai Street, Chaoyang District, Beijing 100021, China.

Received: 20 November 2017 Accepted: 9 April 2018

Published online: 16 April 2018

\section{References}

1. Zhang XM, Fan H, Kou JT, Zhang XX, Li P, Dai Y, et al. Resection of portal and/or superior mesenteric vein and reconstruction by using allogeneic vein for pT3 pancreatic cancer. J Gastroenterol Hepatol. 2016;31:1498-503.

2. Qi Q, Zhuang L, Shen Y, Geng Y, Yu S, Chen H, et al. A novel systemic inflammation response index (SIRI) for predicting the survival of patients with pancreatic cancer after chemotherapy. Cancer. 2016;122:2158-67.

3. Uesaka K, Boku N, Fukutomi A, Okamura Y, Konishi M, Matsumoto I, et al. Adjuvant chemotherapy of S-1 versus gemcitabine for resected pancreatic cancer: a phase 3, open-label, randomised, non-inferiority trial (JASPAC 01). Lancet. 2016;388:248-57.

4. Baker $\mathrm{H}$. Surveillance benefit for pancreatic cancer in high-risk individuals. Lancet Oncol. 2016;17:e227.

5. Vasen H, Ibrahim I, Ponce CG, Slater EP, Matthai E, Carrato A, et al. Benefit of surveillance for pancreatic Cancer in high-risk individuals: outcome of longterm prospective follow-up studies from three European expert centers. J Clin Oncol. 2016;34:2010-9.

6. Chen W, Zheng R, Baade PD, Zhang S, Zeng H, Bray F, et al. Cancer statistics in China, 2015. CA Cancer J Clin. 2016;66:115-32

7. Wolfgang CL, Herman JM, Laheru DA, Klein AP, Erdek MA, Fishman EK, et al. Recent progress in pancreatic cancer. CA Cancer J Clin. 2013;63:318-48.

8. Moore GE, Sako Y, Thomas LB. Radical pancreatoduodenectomy with resection and reanastomosis of the superior mesenteric vein. Surgery. 1951;30:550-3.

9. Yamada S, Fujii T, Sugimoto H, Nomoto S, Takeda S, Kodera Y, et al. Aggressive surgery for borderline resectable pancreatic cancer: evaluation of National Comprehensive Cancer Network quidelines. Pancreas. 2013;42:1004-10.

10. Giovinazzo F, Turri G, Katz MH, Heaton N, Ahmed I. Meta-analysis of benefits of portal-superior mesenteric vein resection in pancreatic resection for ductal adenocarcinoma. Br J Surg. 2016;103:179-91.

11. Yu XZ, Li J, Fu DL, Di Y, Yang F, Hao SJ, et al. Benefit from synchronous portal-superior mesenteric vein resection during pancreaticoduodenectomy for cancer: a meta-analysis. Eur J Surg Oncol. 2014;40:371-8.

12. Murakami Y, Satoi S, Motoi F, Sho M, Kawai M, Matsumoto I, et al. Portal or superior mesenteric vein resection in pancreatoduodenectomy for pancreatic head carcinoma. Br J Surg. 2015;102:837-46.

13. Han SS, Park SJ, Kim SH, Cho SY, Kim YK, Kim TH, et al. Clinical significance of portal-superior mesenteric vein resection in pancreatoduodenectomy for pancreatic head cancer. Pancreas. 2012;41:102-6.

14. Bockhorn M, Uzunoglu FG, Adham M, Imrie C, Milicevic M, Sandberg AA, et al. Borderline resectable pancreatic cancer: a consensus statement by the international study Group of Pancreatic Surgery (ISGPS). Surgery. 2014;155: 977-88.

15. Bassi C, Marchegiani G, Dervenis C, Sarr M, Abu Hilal M, Adham M, et al. The 2016 update of the international study group (ISGPS) definition and grading of postoperative pancreatic fistula: 11 years after. Surgery. 2017;161:584-91.

16. Varadhachary GR, Tamm EP, Abbruzzese JL, Xiong HQ, Crane CH, Wang H, et al. Borderline resectable pancreatic cancer: definitions, management, and role of preoperative therapy. Ann Surg Oncol. 2006;13:1035-46.

17. Conroy T, Bachet JB, Ayav A, Huguet F, Lambert A, Caramella C, et al. Current standards and new innovative approaches for treatment of pancreatic cancer. Eur J Cancer. 2016;57:10-22.

18. Dasari BV, Pasquali S, Vohra RS, Smith AM, Taylor MA, Sutcliffe RP, et al. Extended versus standard lymphadenectomy for pancreatic head Cancer: meta-analysis of randomized controlled trials. J Gastrointest Surg. 2015;19: $1725-32$. 
19. Wang, S.-Y., Chen, W.-L., Wang, Z.-C., et al. High expression of FAMLF is associated with clinical response in patients with de novo acute myeloid leukemia. Leuk. Lymphoma 2016. https://doi.org/10.3109/10428194.2016. 1160087. Leuk Lymphoma. 2017;58:505.

20. Zhou Y, Zhang Z, Liu Y, Li B, Xu D. Pancreatectomy combined with superior mesenteric vein-portal vein resection for pancreatic cancer: a meta-analysis. World J Surg. 2012;36:884-91.

21. Bockhorn M, Burdelski C, Bogoevski D, Sgourakis G, Yekebas EF, Izbicki JR. Arterial en bloc resection for pancreatic carcinoma. Br J Surg. 2011;98:86-92.

22. Ramacciato G, Nigri G, Petrucciani N, Pinna AD, Ravaioli M, Jovine E, et al. Pancreatectomy with mesenteric and portal vein resection for borderline Resectable pancreatic Cancer: multicenter study of 406 patients. Ann Surg Oncol. 2016;23:2028-37.

23. Wang WL, Ye S, Yan S, Shen Y, Zhang M, Wu J, et al. Pancreaticoduodenectomy with portal vein/superior mesenteric vein resection for patients with pancreatic cancer with venous invasion. Hepatobiliary Pancreat Dis Int. 2015;14:429-35.

24. Kim PT, Wei AC, Atenafu EG, Cavallucci D, Cleary SP, Moulton CA, et al. Planned versus unplanned portal vein resections during pancreaticoduodenectomy for adenocarcinoma. Br J Surg. 2013;100:1349-56.

25. Ravikumar R, Sabin C, Abu Hilal M, Bramhall S, White S, Wigmore S, et al. Portal vein resection in borderline resectable pancreatic cancer: a United Kingdom multicenter study. J Am Coll Surg. 2014;218:401-11.

Ready to submit your research? Choose BMC and benefit from:

- fast, convenient online submission

- thorough peer review by experienced researchers in your field

- rapid publication on acceptance

- support for research data, including large and complex data types

- gold Open Access which fosters wider collaboration and increased citations

- maximum visibility for your research: over $100 \mathrm{M}$ website views per year 